\title{
LIDERAZGOS MÁS EFECTIVOS EN LAS EMPRESAS DESARROLLADORAS DE TECNOLOGÍA DE CLASE MUNDIAL
}

\author{
Jesús David Argueta Moreno, Universidad Nacional Autónoma de Honduras (UNAH), \\ Facultad de Ciencias Económicas (FCE), \\ Instituto de Investigaciones Económicas y Sociales (IIES-UNAH), \\ Ciudad Universitaria, Edificio C2, primer piso, Tel/Fax: 504-2239-1849 \\ E-mail: jargueta@iies-unah.org
}

\section{RESUMEN}

Este abordaje empírico de tipo cualitativo, contempla de forma descriptiva, el análisis del rendimiento del Capital Humano en relación al tipo de liderazgo ejercido en las empresas desarrolladoras de tecnología de clase mundial, con la finalidad de determinar qué tipo de metodología gerencial (tipo de liderazgo) resulta más eficiente y eficaz en términos de utilidades (a través del $\mathrm{I}+\mathrm{D}+\mathrm{i}$ ).

Esta investigación se estructuró en 3 segmentos importantes:

E\&A

IIES

29

- El estudio de "Como Dirigir Equipos Virtuales", análisis de Holger Ernst y Martin Hoegl de Harvard Deusto Business Review.

- El análisis de la Teoría Transformacional, de Bernard Bass y Burns.

- El contraste de los rasgos entre los líderes clave, de las Empresas Virtuales en el mundo (Apple, Google, Microsoft y Facebook).

Los cuales se presentarán articulados de forma tal, los tipos de liderazgos imperan en las empresas de corte mundial en este sector, así como del tipo de liderazgo, que resulta más provechoso para las organizaciones de esta magnitud.

El resultado de esta investigación, se espera fomente el análisis de futuras optimizaciones, enfocadas hacia del capital humano en las empresas de corte mundial, en el rubro virtual.

Palabras Clave: Liderazgo, Competitividad, Comunicación. 


\title{
EFFECTIVE LEADERSHIP UPON WORLD CLASS IT COMPANIES
}

Vol. 4

E\&A

IIES

Jesús David Argueta Moreno, Universidad Nacional Autónoma de Honduras (UNAH), Facultad de Ciencias Económicas (FCE), Instituto de Investigaciones Económicas y Sociales (IIES-UNAH), Ciudad Universitaria, Edificio C2, primer piso, Tel/Fax: 504-22391849

E-mail: jargueta@iies-unah.org

\begin{abstract}
This empirical qualitative approach, provides an holistic description of the human capital Engineering performance enhancement in relation to the World Class Companies competitiveness, in order to determine the best trends on the IT market (type of leadership leadership).
\end{abstract}

This research was divided into 3 major segments:

- The study of "Virtual Teams as Management segments" a Holger Ernst and Martin Hoegl analysis, recorded by the Harvard Deusto Business Review.

- Analysis of the Transformational Theory developed by J. Burns and Bernard Bass.

- The contrasting traits among key leaders of Virtual Enterprises in the world (Apple, Google, Microsoft and Facebook).

By identifying the best managerial techniques upon the IT enterprises, applied by the most recognize CEO's on the globe.

The result of this research, contemplates the Human Capital Engineering enhancement through different leadership trends upon the IT World Class Companies.

Keywords: Leadership, Competitiveness and Communication. 


\section{ANTECEDENTES}

El rápido empuje de la globalización, entorno a los distintos sectores productivos $\mathrm{y}$ económicos de las naciones, dicta con suma precisión, las diversas características de gestión y supervivencia de las empresas, según su tamaño, nivel tecnológico, estructura organizacional o grado de dispersión, tanto nivel operativo, funcional y gerencial. Por tanto, si evaluamos la siguiente proporción:

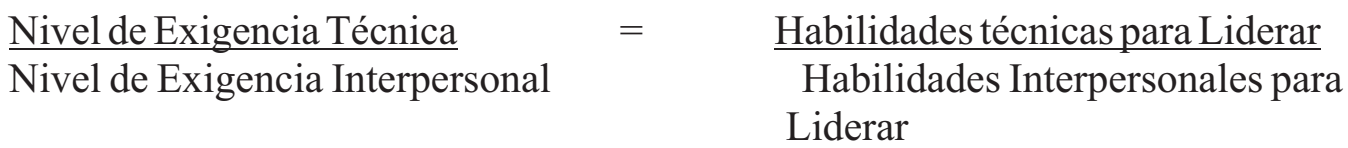

Podemos inferir, que a mayores necesidades técnicas e interpersonales, mayores serán las destrezas, competencias y pericias de liderazgo requeridas para atender estos requerimientos. Destacando que el fenómeno de liderazgo, pasa por la velocidad y capacidad de respuesta gerencial de los líderes en turno, así como en la permeabilidad de sus estilos de dirección según la(s) contingencia(s) por afrontar (Woodward, 1959).

La problemática de las organizaciones desarrolladoras de tecnología en la actualidad, presenta una acentuada tendencia hacia el reproceso y devaluación del capital humano demandado, en respuesta a los estadísticos alarmantes, que documentan sus unidades de contratación y recurso humano, tras identificar las deficiencias técnicas y personales, de su personal (al momento de evaluar sus curvas de rendimiento y desempeño en los distintos campos de acción), los cuales, invitan a un fortalecimiento inmediato, previo a la integración formal a sus labores, situación que de forma superpuesta también requiere de esfuerzos financieros, logísticos y humanos adicionales, para el estímulo de dicho fortalecimiento, el cual se espera presente resultados positivos a mediano y largo plazo, dadas las limitantes manifiestas en la cadena de valor y suministro de la educación superior(Ortega, 2012).

Otro elemento a destacar, respecto a las cadenas de valor de los mercados laborales, responde a la rigidez operativa del profesional contemporáneo, pues estos (en una proporción significativa) no cuentan con la flexibilidad técnica y personal suficiente, para adaptarse a ecosistemas de trabajo ajenos al acostumbrado, por tanto, la crisis del mercado laboral mundial, obliga a los nuevos profesionales, a compatibilizar sus "modus operandi", creando una cultura "camaleónica" de trabajo. Exigiendo una capacidad de respuesta superior al del

E\&A IIES 
Vol. 4

E\&A

IIES

resto de los sectores de gestión, dado el dinamismo y constante fluctuación de su sector.

Por lo anterior, podemos citar que todo líder/gerente/director de una unidad de trabajo, en cualquiera de los niveles jerárquicos al que este asignado, deberá de ser capaz de acentuar su ventaja competitiva y por ende destacarse entre aquellos competidores inmediatos al exponer de forma manifiesta sus competencias tacitas y explicitas, al explorar los distintos niveles de liderazgo de parte de sus superiores, al igual que hacia sus subordinados, a modo de encontrar una zona de confort que les permita desarrollar sus habilidades, pericias y competencias a plenitud.

La asociación del éxito empresarial con los tipos de liderazgo ejercidos, ha sido fuertemente estudiada por las distintas escuelas del pensamiento, particularmente la impulsada por Max Weber (Escuela Estructuralista y Burocrática), sin embargo para fines de este análisis, el enfoque recaerá sobre aquellas inferencias emitidas por los sociólogos Bernard Bass, Holger Ernst y Martin Hoegl, quienes plantean, la existencia de 3 tipos de Líderes (Autocrático, Democrático y Participativo) sobre una masa crítica de subordinados dispersos, en distintas localidades, departamentos, plantas, islas de trabajo, etc. donde en la medida en que se aplique cualquiera de las técnicas de liderazgo mencionadas, se observara un rendimiento especifico (ver anexo No. 1).

\section{EVOLUCIÓN DEL CONOCIMIENTO ENTORNO AL LIDERAZGO}

Previo a cualquier análisis o inferencia de tipo descriptivo, respecto a la variable denominada "Liderazgo", es necesaria la adecuada conceptualización y aproximación teórica del termino madre de este estudio, cuya etimología deriva del inglés "Lead", definido como la acción de encaminar o guiar una actividad, proceso o sistema, con el propósito de alinear un conjunto de esfuerzos y materializarles en el cumplimiento uno o más objetivos. Sin embargo, el "arte/forma" de liderar, es un elemento sobre el cual muchos expertos coluden y divergen, a raíz de su aplicación, experiencias personales, contexto, óptica de las exigencias personales, etc. (Mass, 1990). Por lo anterior, podemos citar, que la tecnología, investigación e innovación, en nombre de la ciencia, han mutado de forma gradual, con la finalidad de estructurar y ejecutar las teorías administrativas a lo largo de la historia, construyendo un caudal de apartados que se vierten en las líneas de investigación de sus cadenas de valor y suministro, respecto al 
conocimiento científico-empresarial. A continuación se adjuntan en la Tabla No. 1, las principales teorías de liderazgo que ejercen un impacto inmediato sobre la gestión del recurso humano.

Tabla No. 1

Línea de Tiempo Evolución del Concepto de Liderazgo

\begin{tabular}{|l|l|l|l|}
\hline Evolución de las Teorías & Autores & Periodo & Factores determinantes \\
\hline Teoría del Gran Hombre & M. Bass & $1920-1950$ & $\begin{array}{l}\text { Características Estables de los } \\
\text { lideres }\end{array}$ \\
\hline $\begin{array}{l}\text { Iniciación } \\
\text { Estructura/Consideración }\end{array}$ & $\begin{array}{l}\text { Larson, Hunt } \\
\text { \& Osborn }\end{array}$ & $1950-1960$ & LDDQ/SBD \\
\hline Teoría de la Contingencia & Fielder & $1967-1978$ & $\begin{array}{l}\text { Estructura de las Tareas/Poder } \\
\text { del Líder/relación líder-seguidor }\end{array}$ \\
\hline Teoría de las Metas & $\begin{array}{l}\text { Evans y } \\
\text { House }\end{array}$ & 1971 & $\begin{array}{l}\text { Motivación lideres-seguidores } \\
\text { mediante ejemplo puntuales }\end{array}$ \\
\hline $\begin{array}{l}\text { Teoría de los Sustitutos de } \\
\text { Liderazgo }\end{array}$ & $\begin{array}{l}\text { Kerr } \\
\text { Jermier }\end{array}$ & 1978 & $\begin{array}{l}\text { Liderazgo depende del contexto y } \\
\text { capacidad de sus subordinados }\end{array}$ \\
\hline $\begin{array}{l}\text { Teoría Transformacional } \\
\text { Burns }\end{array}$ & 1978 & $\begin{array}{l}\text { Liderazgo contagia a seguidores, } \\
\text { carisma, inspiración, } \\
\text { estimulación intelectual y } \\
\text { consideración individualizada. }\end{array}$ \\
\hline $\begin{array}{l}\text { Teoría de los Recursos } \\
\text { Cognitivos }\end{array}$ & $\begin{array}{l}\text { Fiedler } \\
\text { García }\end{array}$ & $\begin{array}{l}\text { Surge el Liderazgo de tipo } \\
\text { carismático (MLQ) }\end{array}$ \\
\hline Avecnológico del Liderazgo & Bodge \& & $\begin{array}{l}\text { Evalúan la relación rendimiento- } \\
\text { estrés interpersonal-complejidad } \\
\text { de las tareas }\end{array}$ \\
\hline
\end{tabular}

Fuente: S. Rego 1998

En la Tabla anterior, apreciamos una línea de tiempo, que detalla la evolución del liderazgo orientado hacia el estímulo del Capital Intelectual en el recurso humano. En esta tabla destaca la Teoría de Contingencia de Fiedler, La Teoría de las Metas Evans, la Teoría Transformacional de Bernard Mass y la Teoría del Liderazgo Tecnológico, las cuales en su recorrido, convergen en un formato gerencial de control de la gestión denominado "E-Leadership". El presente documento, tiene la característica de validar a través del método científico, que la evolución ultima del liderazgo entre los CEO's de las Empresas desarrolladoras de tecnología de Clase Mundial, apunta hacia esa tendencia con la finalidad de generar un dinamismo mayor en el clima de investigación, desarrollo e innovación 
Vol. 4

E\&A

IIES

entre las distintas organizaciones.

Cabe mencionar, que en esta tabla no se adiciono a la teoría transaccional, dado que la teoría transformacional, representa una optimización de la misma, incluyendo un sin número de componentes adicionales, que más haya de contemplar elementos de liderazgo correctivo, evalúan la aplicación de una nueva tendencia gerencial en pro del buen desempeño laboral de sus asociados (Zacarro, 2001), a continuación se presentaran las 2 posturas principales, desarrolladas por la teoría transformacional:

- El líder motiva a un número determinado de seguidores a lo interno de sus unidades de trabajo, los cuales se ven inspirados por el mismo, generando un efecto multiplicador en la organización.

- El líder, adquiere una postura transitoria, el cual deberá dejar un legado organizacional, de procesos y rendimiento, mediante el estímulo de los valores socioemocionales e intelectuales de los asociados a su cargo.

En aras de responder a la interrogante planteada con anterioridad, se ha contemplado el abordaje del Modelo propuesto por Hogel Ernst y Martin Hoegl, en su estudio efectuado en el año 2012, denominado "Como Dirigir Equipos Virtuales", al retomar la temática de Bernard Mass sobre los tipos de liderazgo predominantes en las empresas de clase mundial, acondicionando esta concepción, a las empresas de orden tecnológico.

De igual forma, en el Anexo No. 2, Figura No.1, podemos destacar que el modelo de línea base de esta investigación, se centra en la descripción de las 3 técnicas de liderazgo clave (Mass, 1990), como variables independientes del constructo y su impacto, en el rendimiento del Capital Humano.

\section{DESCRIPCIÓN DEL MODELO DE LÍNEA BASE APLICADO A LA INVESTIGACIÓN}

Como se había señalado de forma general con anterioridad. El modelo de línea base de este análisis, se basara en el conocimiento creado por los sociólogos, Bernand Mass, Holger Ernst y Martin Hoegl, en su investigación "Como Dirigir Equipos Virtuales", el cual responde a un estudio de cohorte, sobre 80 equipos de desarrollo de software alrededor de todo el mundo (Laboratorios ubicados en Brasil, China, Francia, Dinamarca, India y Estados Unidos), estos laboratorios en análisis, variaron en tamaño de 20 - 5,000 desarrolladores, donde cada equipo de trabajo fue conformado por hasta 9 miembros . El estudio de Ernst y Hoegl, abarco 
el análisis de 392 líderes de grupo, de los 80 laboratorios en gestión. Esta misma propuesta, contemplo la creación de un índice de rendimiento en términos de la eficacia de los equipos respecto a:

- Calidad de sus productos

- Fiabilidad de los procesos de supervisión y auditoria

- Satisfacción del Cliente

- Utilidad

- Etc.

¿Qué tipos de Empresas participaron en el análisis de Hoegel y Ernst? En el desarrollo de la propuesta de los sociólogos Hoegel y Ernst, participaron empresas de la magnitud de IBM, SAP, General Electric (GE) entro otros, quienes por su prestigio y alcance corporativo, otorgan validez al instrumento de medición, al contar con un 0.89 de intensidad (George y Malllery en 1995, criterios de aceptación Alfa de Crombach), denotando el peso estadístico de la efectividad y precisión de su instrumento de medición.

De igual forma, Ernst y Hoegl, proponen que el factor clave para la optimización del rendimiento en las empresas desarrolladoras de Tecnología de clase mundial, responden directamente a:

- El estímulo de los procesos críticos de trabajo en equipo (los cuales e acentúan al conformar equipos multidisciplinarios de trabajo) denominados Key Performance Indicators (KPI'S).

- El tipo de liderazgo ejercido en las empresas desarrolladoras de tecnología exitosas, depende a su vez del grado de dispersión sobre las unidades de trabajo subordinadas,

- Los niveles sistematización y burocratización de los roles de trabajo en cada agrupación de empleados.

\section{CONTRASTE DE LOS RASGOS ENTRE LOS LÍDERES CLAVE, DE LAS EMPRESAS VIRTUALES EN EL MUNDO (APPLE, GOOGLE, MICROSOFTY FACEBOOK)}

En este apartado hemos de identificar aquellos rasgos de liderazgo que más se acentúan sobre 4 de los directivos en el ámbito de la Investigación, Desarrollo e Innovación $(\mathrm{I}+\mathrm{D}+\mathrm{i})$, que han marcado época en sector de desarrollo de tecnología, como ser: 
Vol. 4

E\&A

IIES
- Steve Jobs

- Bill Gates

- Eric Schmidt

- Mark Zuckerberg

Los Directivos antes mencionados, cambiaron la óptica de la tecnológica con sus innovaciones y desarrollo tecnológico, los cuales a través de sus virtudes técnicas y genéricas, definen escenarios futuros, mediante instrumentos de pronósticos hechos por ellos mismos, los cuales les permitieron anticiparse a los posibles impactos o efectos de sus productos en el mercado, con suma precisión en sus proyecciones.

Los Gurús del "management" como Peter Drucker y Ken Blanchard coinciden en diversos artículos en que una característica diferencial de los líderes transformadores, respecto a un directivo o profesionista "tradicional", es su enfoque en el cambio. Pues este tipo de personas busca "crear visión" y luego trabajar para que sus equipos se orienten a la transformación (Andersen, 2002).

Steve Jobs por ejemplo, fue una figura enigmática, con innegables dotes de visión; en los cuales demostró ser capaz de llevar al mercado plataformas y productos que son el resultado de una anticipación de necesidades. Este rasgo único de Steve Jobs, fue estudiado con suma meticulosidad por la Psicóloga Industrial Patricia González (Docente UDLAP y Tecnológico de Monterrey, México), quien complemento el planteamiento desarrollado por su homologo Lorenzo Piñón (Docente del Tecnológico de Monterrey, México), citando que "En la actualidad se vive un nuevo paradigma donde la gente tiene más capacidad de decisión y desea una experiencia de consumo mayor. A la par, las personas experimentan la presión de tener que diferenciarse si desean una mejor proyección, y es un hecho que no todos los jefes o profesionistas llegan a ser líderes; hay quienes sólo serán buenos para gestionar, lo que no implica que delinearán cambios". Por tanto, para ser un empresario/Líder con desempeño sobresaliente, conviene apelar al estímulo de las potencialidades del Capital Humano y el contar con cierto grado aversión al riesgo, destacando entre ellas, las pericias manifiestas de los gestores de Apple, Google, Facebook y Microsoft que pueden ser replicadas por cualquier gerente en busca de un giro a su trayectoria.

El docente Lorenzo Piñón, señala que aparte de la característica antes mencionada, se pueden destacar 6 rasgos comunes, entre los máximos líderes de las Empresas Virtuales de Clase Mundial, que marcan la diferencia y representan pilares 
fundamentales para el éxito profesional de estos, los cuales se presentan a continuación:

1. Escucha opiniones. Steve Jobs realizaba sesiones trimestrales donde cada integrante de su equipo debe exponer los problemas y avances que ha tenido en el desarrollo de algún proyecto (contradiciendo el prototipo autocrático que suele criticársele en su modelo primario de negocios). Proporcionando distintas conferencias alrededor del globo, para llenar de 'energía' a sus asociados y equipos trabajo; promoviendo una cultura de tomar riesgos (Gallo, 2009).

2. Reto. Según el Ex CEO de Google, Eric Schmidt, cualquiera puede ser el "autor" de una idea sobresaliente en el trabajo, sin embargo, para llevarla a cabo, este individuo necesitará del apoyo y el trabajo de otros, así que es importante el contagiar a quienes se encuentran involucrados con esa visión. Además, los proyectos destacados no siempre se le ocurren a la misma persona, las ideas buenas pueden provenir de un grupo y hay que prestar atención a ellas (Schmidt, 1982).

3. Fuera conformidad. Otro rasgo del creador del "iPad" fue su obsesión por los detalles y su "cero tolerancia hacia la mediocridad", menciona Piñón, agregando, que pese a que Steve Jobs gustaba de tener todo bajo control, "aprendió de sus errores y meditó sobre la obtención de un producto perfecto". La meta del líder de la tecnología es hacer "la vida de alguien más elegante y fácil" (Schmidt, 1982).

4. Destacarse. Según Bill Gates, el sobresalir implica esforzarse a diario por hacer bien el trabajo, y no culpar a otros porque no se aplicó, todo lo que se debía, para dar los resultados esperados (Relatado en las investigaciones de Gabriela Lorenzo (Docente Investigadora del Tecnológico de Monterrey). Por otro lado, el principio básico para tener un alto desempeño según Steve Jobs, es amar lo que hacemos. Jobs alguna vez dijo a sus empleados "la gente con pasión puede cambiar el mundo para mejor. Es difícil tener ideas nuevas y creativas, si no eres un apasionado". "Sea que tengamos una carrera corporativa o un negocio propio, lo básico 
Vol. 4

E\&A

IIES

LIDERAZGOS MÁS EFECTIVOS EN LAS EMPRESAS DESARROLLADORAS DE TECNOLOGÍA DE CLASE MUNDIAL

es: observar, entender necesidades y después pensar en estrategias para que tus clientes cumplan, a través de tu trabajo, una meta. Ahora bien, no se trata de entregar por entregar un resultado, hay que buscar destacarlo y venderlo como un sueño" (Gallo, 2009).

5. Vende sueños. Jobs sabía que los seguidores de Apple no son (sólo) consumidores, son personas con ambiciones y sueños (Gallo, 2009).

6. La 'magia' del no. Steve Jobs señalo en algunas conferencias: "Estoy tan orgulloso de lo que no hacemos como de lo que hacemos". Comprometiéndose a crear productos con un diseño más simple y ordenado. Pues en el mundo de Apple, la innovación significó eliminar lo innecesario.

Una vez definido el campo en el cual, la empresa se pretende diferenciar, es trascendental la creación de un plan de desarrollo y gestión, así como de la inmediatez de los recursos para hacerlo. Aunque existan 'tentaciones' para desertar ese deseo a incursionar en otros terrenos, el mantenerse firme puede asegurar que la meta se cumpla.

Tabla No. 2

Características Principales de los líderes con mayor impacto en la época contemporánea

\begin{tabular}{|l|c|l|l|c|}
\hline Nombres & Steve Jobs & Bill Gates & Eric Schmidt & Mark Zuckerberg \\
\hline Marcas & Apple & Microsoft & Google & Facebook \\
\hline Rasgos de Liderazgo & \multicolumn{4}{|l|}{} \\
\hline Escucha Opiniones & $\mathrm{X}$ & & $\mathrm{X}$ & $\mathrm{X}$ \\
\hline La gusta sumir Retos & $\mathrm{X}$ & & $\mathrm{X}$ & $\mathrm{X}$ \\
\hline Fuera de Conformidad & $\mathrm{X}$ & $\mathrm{X}$ & $\mathrm{X}$ & $\mathrm{X}$ \\
\hline $\begin{array}{l}\text { Se Destaca/AItamente } \\
\text { Competitivo }\end{array}$ & & $\mathrm{X}$ & & $\mathrm{X}$ \\
\hline Vende Sueños & $\mathrm{X}$ & & $\mathrm{X}$ & $\mathrm{X}$ \\
\hline $\begin{array}{l}\text { Acepta y se Compromete } \\
\text { a mitigar el Riesgo desde } \\
\text { distintos frentes }\end{array}$ & $\mathrm{X}$ & $\mathrm{X}$ & $\mathrm{X}$ & $\mathrm{X}$ \\
\hline Tipo de Liderazgo & Autocrático & Democrático & Democrático & Participativo \\
\hline
\end{tabular}

Fuente: Revista Summa, Edición Junio 2012 y HSM

2 http://appleweblog.com/2011/10/las-30-mejores-frases-y-citas-de-steve-jobs, Frases Celebres del Líder de APPLE Steve Jobs 
Cabe mencionar que tras evaluar a 4 de los principales líderes de las empresas desarrolladoras de tecnología en la actualidad, vemos que la tendencia se inclina hacia un hibrido de liderazgo democrático, fuera de conformidad y capaz de aceptar el riesgo venidero mostrando elementos de estímulo motivacionalintelectual y Estratégico-Innovador (Bass, Transformational and Transactional Leadership, 1998).

\section{EL IMPACTO DE LA TEORÍA TRANSFORMACIONAL EN EL LIDERAZGO}

La evolución de la teoría transformacional y su concepto de liderazgo ha trascendido en su óptica e interpretación del mismo, más allá del enfoque transaccional, el cual en su momento presento cierta rigidez en los roles y funciones de los asociados subordinados, en relación a su director más inmediato, sin embargo este modelo transformacional engloba una versión de liderazgo, encaminada hacia el gerenciamiento preventivo de anomalías operativas, destacando 4 pilares sobre los cuales descansa este modelo, expuestos a continuación:

1. Influencia Idealizada (Liderazgo Carismático): Este es fuerte entre líderes que tienen una visión y sentido de misión; ganando el respeto, confianza y seguridad de sus subordinados; y que adquieren una identificación individual fuerte de sus seguidores. Los líderes que presentan influencia idealizada son capaces de obtener el esfuerzo extra requerido de los seguidores para lograr niveles óptimos de desarrollo y desempeño.

2. Consideración Individualizada. Los líderes se concentran en diagnosticar las necesidades y capacidades de los seguidores. Al identificar y atender las necesidades de sus seguidores individualmente. También delegan, entrenan, aconsejan y proveen retroalimentación para el uso en el desarrollo personal de los seguidores y con ello, elevando el nivel de necesidad y seguridad de sus seguidores para adquirir mayores niveles de responsabilidad.

3. Estimulación Intelectual: Los líderes fomentan activamente la creatividad, y enfatizan un re-pensamiento y re-examinación de suposiciones subyacentes a los problemas, utilizando la intuición así como una lógica más formal para solucionar los problemas. Ocasionando que los seguidores se transformen en solucionadores de problemas más efectivos con y sin la facilitación del líder.

4. Liderazgo Inspiracional: Los líderes dan ánimo, aumentan el optimismo y entusiasmo, y comunican sus visiones de futuros realizables con fluidez y

E\&A IIES 
Vol. 4

E\&A

IIES

seguridad. Proveen visión la cual estimula la energía para lograr altos niveles de desempeño y desarrollo.

Finalmente, hay varias maneras de ser transformacional. El líder carismático puede infundir un sentido de misión, especialmente en situaciones de alta ansiedad, condiciones de crisis y cambio, que intensifican los procesos de protección, transferencia y atribución; de igual forma, el líder que es considerado por los individuos puede elevar los intereses de los subordinados a intereses de mayor nivel; el líder que estimula intelectualmente puede articular una visión compartida de posibilidades conjuntas aceptables.

Sin embargo, Pese al éxito en la aplicación de esta teoría en el ámbito financiero-tecnológico, es vital que se enumeren los puntos sobre los cuales recaen las fortalezas y debilidades del modelo transformacional en el sector virtual, detallado a continuación en la tabla No. 3 .

Tabla No. 3

Análisis de la Teoría Transformacional

\begin{tabular}{|c|c|}
\hline $\begin{array}{l}\text { Fortalezas de la Teoría } \\
\text { Transformacional }\end{array}$ & Debilidades de la Teoría Transformacional \\
\hline $\begin{array}{l}\text { El liderazgo emerge de la } \\
\text { relación lideres seguidores }\end{array}$ & Le falta claridad conceptual en su desarrollo \\
\hline $\begin{array}{l}\text { Trabaja con la dimensión } \\
\text { ética }\end{array}$ & $\begin{array}{l}\begin{array}{l}\text { Algunos tachan el modelo de elitista } \\
\text { antidemocrático }\end{array} \\
\text { y }\end{array}$ \\
\hline $\begin{array}{l}\text { Tiene en cuenta las } \\
\text { necesidades y desarrollo de } \\
\text { los seguidores }\end{array}$ & $\begin{array}{l}\text { Puede generar conductas de abuso de poder, por } \\
\text { parte del líder }\end{array}$ \\
\hline $\begin{array}{l}\mathrm{Su} \text { Implementación ha } \\
\text { demostrado ser exitosa en la } \\
\text { mayoría de los casos }\end{array}$ & $* * * * * * * * * * * * * * * * * *$ \\
\hline
\end{tabular}

Fuente: Interpretación Personal

Luego de citar las fortalezas y debilidades del modelo, es vital hacer mención, que la teoría transformacional, persigue la aplicación de elementos éticos intagibles/abstractos, hacia la aplicabilidad diaria conjunta de los grupos/equipos de trabajo, clasificando a estos componentes socioemocionales en dimenciones, las cuales se iran cumpliendo según el tipo de liderazgo aplicado e intensidad en la sistematización de los procesos (ver anexo No. 4, Figura No.3). 


\section{INTERPRETACIÓN DE LOS DATOS RECABADOS POR LA INVESTIGACIÓN DE ERNST A ESTE ANÁLISIS}

El estudio de Ernst, evaluó a un total de 392 directivos (líderes de equipo) que participaron en su estudio, procurando evaluar a una masa crítica de líderes significativa, con el fin de garantizar la validez de los resultados y así superar el habitual sesgo del método estadístico de recopilación de información.

Tras revisar de forma holística la investigación de Ernst y adentrarnos en la descripción de los datos obtenidos, percibimos en sus análisis, los procesos sistémicos de trabajo en equipo, se pueden clasificar en dos categorías:

1. Las Tareas Relacionadas (entre ellas, las que contribuyen a garantizar que todos los miembros están plenamente comprometidos).

2. Socios emocionales (entre ellos, los que incrementan la cohesión del grupo).

Este análisis encaminado por Martin Hoegl, indica que aquellos procesos que estén directamente relacionados con las tareas críticas de los procesos sistémicos (funcionales, operacionales o gerenciales) en los equipos de trabajo virtuales, son los cruciales para elevar, mantener, medir o controlar el rendimiento de los equipos dispersos.

En concreto, los equipos virtuales que cuenten con procesos que estimulen y potencien el apoyo mutuo, sinergias y coordinación de esfuerzos entre los miembros de cada unidad de trabajo, equilibrio de las aportaciones y comunicación relacionada con las tareas, superaran a otros equipos con grados de cohesión más bajos (hasta equipos de trabajo presenciales). Además, los equipos dispersos que posean un alto número de procesos relacionados con las tareas fueron capaces de superar de forma significativamente a los equipos presenciales con un número similar de esos mismos procesos, a pesar de la separación física de sus miembros. En otras palabras, el efecto global de la dispersión no es necesariamente perjudicial, sino que depende de la calidad de los procesos relacionados con las tareas asignadas a un equipo.

A pesar de esto, la dispersión comporta riesgos destacados: Donde los equipos con deficiente sistematización de los procesos relacionados con las tareas de cohesión y comunicación, se vieron afectados en mayor medida por el aumento de la dispersión. 
Vol. 4

E\&A

IIES

De igual forma, en Anexo 3, Figura No. 2, se aprecia la evolución del fenómeno de dispersión, sobre el rendimiento, en términos de eficiencia y eficacia, quienes, pese a las distintas intensidades de dispersión a las que fueron sometidas, reflejan una considerable mejora, una vez que se fueron incorporados los instrumentos de optimización y mecanización de los procesos/actividades. En esta misma línea, hacemos mención que de los 392 líderes encuestados, un poco más de los $2 / 5$, poseían un tipos de liderazgo participativo, $2 / 5$ un tipo de liderazgo autocrático y $1 / 5$ un tipo de liderazgo democrático, lo que nos indica, que el tipo de liderazgo en las empresas de corte virtual es un hibrido, entre los 3 antes mencionados, recurriendo a una nueva tendencia de gerenciar denominada "E-leadership".

Más allá de los procesos relacionados con las tareas, las organizaciones también deben asegurarse de que los miembros de los equipos estén comprometidos con los objetivos globales, que se identifican con el grupo y que apoyan de forma activa un espíritu de equipo. En otras palabras, los procesos socioemocionales también son importantes (Jimenez, 2005).

\section{CONCLUSIONES}

Tras explorar el sustento teórico respecto a los tipos de liderazgo, así como el abordaje del marco referencial contemporáneo, en el cual se gestan las Empresas Desarrolladoras de Tecnología, se ha procurado la articulación de ambos frentes mediante el planteamiento de Ernst y Hoegl, al determinar el tipo de liderazgo que impera en el sector, sugiriendo la adopción de una percepción globalizada, en mecanismo interconectados denominados "Networks". En consecuencia, es necesario que los directivos y los miembros de los equipos sensibilicen a sus subordinados, respecto al reconocimiento de la naturaleza de las operaciones de la empresa en todos sus niveles (operativo, funcional y estratégico), así como de los mercados sujetados y potenciales de la organización. Por otro lado las empresas desarrolladoras de tecnología como Facebook, Google, Apple y Microsoft -conocidas todas ellas por el alcance global de sus actividades comercialesresponden todas a los mismos patrones de liderazgo estratégico denominado ELeadership. Donde este tipo de prácticas mejora el desarrollo de actitudes abiertas a la diversidad y la capacidad para trabajar en diferentes contextos y contribuye al abordaje rápido de los retos al trabajar en equipos virtuales.

De igual forma a través de esta investigación, se dimensionaron las pericias y aptitudes organizacionales de 4 líderes predominantes y contemporáneos en el sector Desarrollador de Tecnología, a quienes se les categorizo en acorde a sus 
métodos de gestión de la autoridad y liderazgo en el sector. Denotando que entre los rasgos más prominentes encontrados sobre los directores del sector, el tipo de Liderazgo que más predomina es el Democrático, el cual presentan más semejanzas con la nueva tendencia reciente denominada E-leadership (Burke, 2012), la cual tiene la finalidad de agilizar el flujo de los sistemas de producción y manufactura, optimizando de forma paralela, el rendimiento del recurso humano y su interacción con las herramientas tecnológicas de trabajo.

Finalmente, el efecto global de la dispersión, no es necesariamente perjudicial, sino que depende del nivel de sistematización de los procesos relacionados con las tareas críticas de un equipo, así como del tipo de liderazgo que se ejerza, el cual dé lugar a la creación de equipos virtuales, que tomen en cuenta las habilidades sociales y de autosuficiencia de sus miembros, así como de los subordinados a su cargo.

\section{BIBLIOGRAFÍA}

Andersen, A., 2002. Strategic Leadership. Institute for Strategic Change, Editorial III.

Burke, R., 2012. E-Leadership. Metafuture, http://www.metafuture.org/articlesbycolleagues/RobertBurke/eleadership.htm.

Gallo, C., 2009. The Innovation Secrets of Steve Jobs. Denver, Us: Mc Graw Hill.

Jiménez, R., 2005. Trayectoria de la Mujer en las Empresas Familiares. Madrid, España: Universidad de Jaen.

Mass, B., 1990. Leadership and Performance Beyond Expectations. New York, US: The Free Press.

Ortega, C., 2012. Oferta y Demanda de Profesionales: Cadena de Valor y Suministro de la Educación Superior. Tegucigalpa: IIES - UNAH, Proyecto OyD, Revista E\&A.

Schmidt, E., 1982. Controlling Large Software Development in a Controlled Enviorment. Conneticut, US: Berkley University Technical Reports.

E\&A IIES 
Vol. 4

E\&A

IIES

Woodward, J., 1959. Impacto de la tecnología sobre la estructura organizacional empresarial. Reino Unido: Free Press.

Zacarro, S., 2001. The Nature of Executive Leadership, a conceptual and Empirical Analysis of Success. Washington D.C.: American Psycology Asociation.

\section{ANEXOS}

\section{Anexo 1}

\section{Características de los 3 Tipos de Liderazgo}

\section{Liderazgo Autocrático:}

El Estilo autocrático, se define como aquel en que el líder da la orden sin consulta previa y solo espera el cumplimiento. Es dogmático y firme. Dirige mediante la habilidad para dar o negar recompensas y castigos. Es recomendable para resolver situaciones emergentes, o cuando los subalternos evidencian poca madurez laboral y emocional.

\section{Liderazgo Democrático:}

El estilo del líder democrático o participativo se caracteriza por la consulta y la persuasión a sus subordinados en torno a las acciones y decisiones propuestas, $\mathrm{y}$ alienta la participación de los mismos. Es el estilo más recomendable en las labores pedagógicas y administrativas porque, según evidencia empírica, es la que produce un mayor grado de motivación, tan necesario para el aprendizaje.

\section{Liderazgo Participativo:}

El líder participativo utiliza muy poco su poder, si es que lo usa concede a los subalternos un alto grado de independencia en sus tareas. Depende en gran medida de los subalternos para fijar sus propias metas y medios para ellos. Considera que su papel es apoyar las acciones de los seguidores al proporcionarles información y actuando primordialmente como contacto entre él y el grupo. Es útil y recomendable cuando el grupo evidencia un alto grado de madurez, es decir atando son más hábiles y más seguros que el propio líder. 
Anexo No. 2, Figura No.1

Variables en estudio, Modelo de línea base del Análisis

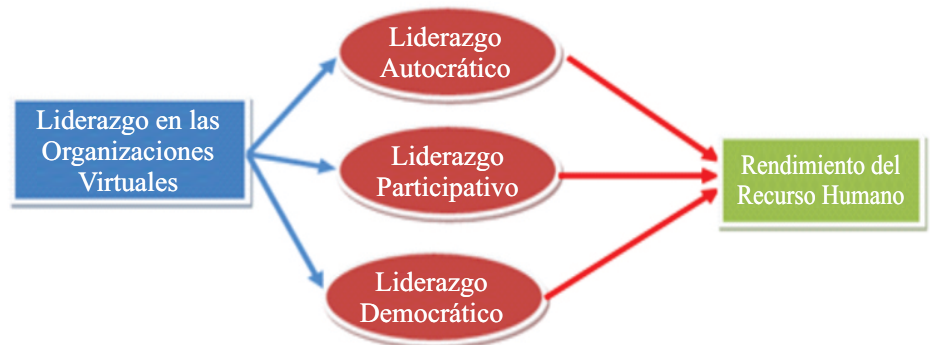

Fuente: Interpretación Personal

\section{Anexo No. 3, Figura No.2}

Dispersión de las tendencias de rendimiento y dispersión:

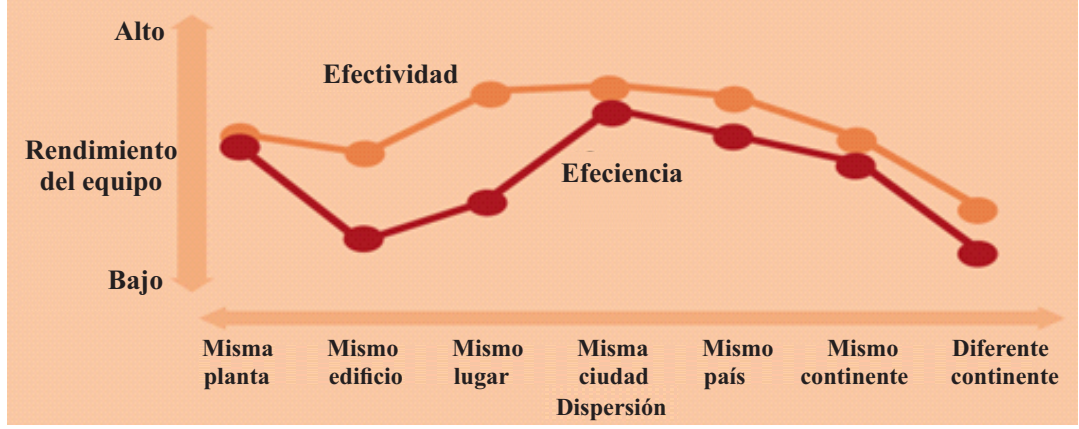

Fuente: Frank Siebdrat y Martin Hoegl, 2010, Harvard Business Review.

\section{Anexo No. 4, Figura No.3}

Dimensiones de la Teoría Transformacional:

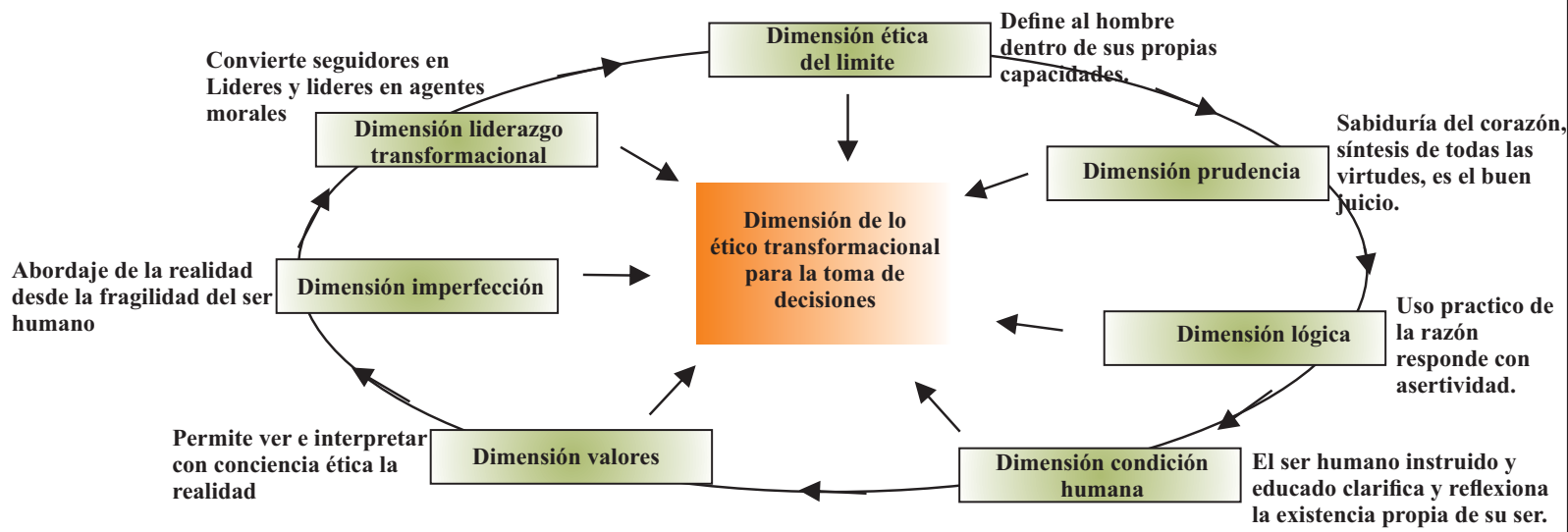

Fuentes: Rocío Jiménez, 2005, "La Trayectoria de las Mujeres en las Empresas Familiares" 